\title{
Value of intraoperative pleural lavage in staging non-small cell lung cancer
}

Thomas A. D'Amico, MD

See related article on page 1113.

From Duke University Medical Center, Durham, NC.

Received for publication Dec 1, 2003; accepted for publication Dec 12, 2003.

Address for reprints: Thomas A. D'Amico, MD, Associate Professor of Surgery, Duke University Medical Center, Box 3496, Durham, NC $27710 \quad$ (E-mail: damic001@mc.duke.edu).

J Thorac Cardiovasc Surg 2004;127:947-8 $0022-5223 / \$ 30.00$

Copyright (C) 2004 by The American Association for Thoracic Surgery

doi:10.1016/j.jtcvs.2003.12.015

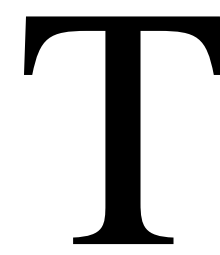

he use of adjunctive staging techniques in patients with potentially resectable non-small cell lung cancer (NSCLC) will improve survival in two ways, by selecting patients for whom surgery would be futile and by selecting patients for whom induction or adjuvant therapy could improve survival. Various biologic staging parameters have been applied to the primary tumor, ${ }^{1}$ lymph nodes, ${ }^{2}$ bone mar-

row, ${ }^{3}$ and serum (Brooks and colleagues, unpublished data) with demonstrated effective stratification in terms of survival and risk of recurrence. Furthermore, analysis of molecular characteristics of the tumor may stratify patients according to response to chemotherapy. ${ }^{4}$ The strategy of pleural lavage cytologic study may accomplish one, both, or neither of these objectives, depending on how it is applied.

The efficacy of pleural lavage cytologic examination was investigated by Lim and colleagues 5 in a series of 292 patients who underwent thoracotomy for lung cancer. In this series, $13(4.5 \%)$ of 292 of patients were found to have positive cytologic results; however, only 5 of the 292 patients had pathologic stage I or II disease (irrespective of the pleural cytologic result), and the remaining patients had disease already classified as stage IIIA (5 patients), stage IIIB ( 2 patients), or stage IV (1 patient). Thus few patients had disease upstaging as a result of this technique. For a staging technique (conventional or biologic) to be considered effective, a significant portion of patients should be affected; it appears that only $1 \%$ to $2 \%$ of patients would have upstaging as a result of pleural cytologic examination.

Positive cytologic results, in addition to pathologic stage, had a negative impact on survival in this series. Unfortunately, the follow-up is incomplete; median follow-up is only 15 months (range 1-40 months). As more patients with early stage disease and negative pleural cytologic results have relapses in time, the stratification effect of positive pleural cytologic results may be diminished. Lim and colleagues ${ }^{5}$ compared the survival of a group of 17 patients with stage IIIB (T4) disease with the patients in the study with positive pleural cytologic results, a comparison that is not germane to their study. Of the 17 patients with stage IIIB disease, 15 of 17 had T4 status defined by the presence of a satellite nodule only. It is well known that the survival in this subgroup is much better than those of the malignant pleural effusion or N3 subgroups.

The goals of preoperative staging are to select patients who would benefit from induction therapy and to select patients for immediate resection. As currently described, pleural lavage performed at the time of thoracotomy will not effectively select patients for induction therapy. Furthermore, this technique will not effectively exclude patients from futile surgery, because a significant component of the risk of surgery is the thoracotomy itself. Patients who undergo futile thoracotomy may suffer in one of the following ways: the inherent risk of the procedure (complications, including death), postoperative pain, delay in receiving chemotherapy, a higher risk of incomplete chemotherapy dosing, and delay or interruption of radiation therapy. However, performance of the procedure preoperatively, either percutaneously or with minimally invasive techniques, combined with real-time cytologic analysis, may make this strategy successful.

Regarding assignment of treatment, there is no evidence that induction or adjuvant therapy would be useful in this population of patients (those with positive pleural cytologic results), but this may be a question for future studies. Currently, 
the role of pleural lavage is being analyzed in two completed multi-institutional trials: Cancer and Leukemia Group B (CALGB) 159902: Markers of Pleural Involvement in NSCLC; and American College of Surgeons Oncology Group (ACOSOG) Z0040: Prognostic Significance of Occult Metastases in NSCLC. In addition, other emerging technologies may improve risk stratification and selection of therapy for patients with potentially resectable NSCLC. Intraoperative assessment of lymph nodes with real-time polymerase chain reaction may improve the ability to detect occult mediastinal lymph node involvement at mediastinoscopy. The development of the sentinel node technique for lung cancer (CALGB 140203) may allow complete molecular analysis of a single lymph node station to determine the degree of nodal involvement.

Stage-specific therapy of NSCLC depends on thorough preoperative and intraoperative staging. Techniques that improve risk stratification may identify subgroups of patients in whom induction or adjuvant therapy is beneficial. Currently, induction therapy is reserved for patients with advanced disease, although ongoing protocols offering induction therapy to a wider range of patients may demonstrate an advantage to that strategy. Recent studies have demonstrated that adjuvant chemotherapy is associated with a marginal (4\%-5\%) increase in survival among patients with completely resected lung cancer (stage I-III). ${ }^{6,7}$ To improve overall survival of patients with NSCLC, we must continue to investigate novel staging and treatment strategies. Surgeons should be intimately involved with the de- sign, execution, and review of these studies to ensure clinical and scientific relevance, clinical quality, and appropriate tissue acquisition.

\section{References}

1. D'Amico TA, Massey M, Herndon JE, Moore MB, Harpole DH. A biologic risk model for stage I lung cancer: immunohistochemical analysis of 408 patients with the use of ten molecular markers. J Thorac Cardiovasc Surg. 1999;117:736-43.

2. D'Cunha J, Corfits AL, Herndon JE, Kern JA, Kohman LJ, Patterson GA, et al. Molecular staging of lung cancer: real-time polymerase chain reaction estimation of lymph node micrometastatic tumor cell burden in stage I non-small cell lung cancer-preliminary results of Cancer and Leukemia Group B Trial 9761. J Thorac Cardiovasc Surg. 2002;123: 484-91.

3. Lau CL, D'Amico TA, Harpole DH. Staging and prognosis: clinical and molecular prognostic factors and models for non-small cell lung cancer. In: Pass HI, Mitchell JB, Johnson DH, Turrisi AT, Minna JD, editors. Lung cancer principles and practice. 2nd ed.. Philadelphia: Lippincott, Williams \& Wilkins; 2000.

4. Brooks KR, To K, Moore MB, Conlon DH, Herndon JE, D'Amico TA, et al. Measurement of chemoresistance markers in patients with stage III non-small cell lung cancer: a novel approach for patient selection. Ann Thorac Surg. 2003;76:187-93.

5. Lim E, Ali A, Theodorou P, Nicholson AG, Ladas G, Goldstraw P. Intraoperative pleural lavage cytology is an independent prognostic indicator for staging non-small cell lung cancer. J Thorac Cardiovasc Surg. 2004;127:1113-8.

6. Non-Small Cell Lung Cancer Collaborative Group. Chemotherapy in non-small cell lung cancer: a meta-analysis using updated data on individual patients from 52 randomised clinical trials. BMJ. 1995;311: 899-909.

7. Le Chevalier, IALT Investigators. Results of the Randomized International Adjuvant Lung Cancer Trial (IALT): cisplatin-based chemotherapy (CT) vs no CT in 1867 patients (pts) with resected non-small cell lung cancer (NSCLC). Proc Am Soc Clin Oncol. 2003;22:2. 